\title{
The Effect of High-Frequency Repetitive Transcranial Magnetic Stimulation on Occupational Stress among Health Care Workers: A Pilot Study
}

\author{
Young In Kim¹, Sun Mi Kim ${ }^{1}$, Hyungjin $\mathrm{Kim}^{2}$, and Doug Hyun Han ${ }^{1}$ \\ 1Department of Psychiatry, Chung-Ang University Hospital, Seoul, Republic of Korea \\ ${ }^{2}$ Department of Psychology, Rice University, Houston, TX, USA
}

\begin{abstract}
Objective Repetitive transcranial magnetic stimulation (rTMS) was approved by the Food and Drug Administration to alleviate symptoms of treatment-resistant depression. This study aimed to evaluate the effectiveness of rTMS treatment on alleviating occupational stress by evaluating clinical symptoms and quantitative electroencephalography (QEEG).

Methods Twenty-four health care workers were randomized to receive 12 sessions of active or sham rTMS delivered to the left dorsolateral prefrontal cortex (DLPFC). Each session consisted of 32 trains of $10 \mathrm{~Hz}$ repetitive TMS delivered in 5-second trains at $110 \%$ of the estimated prefrontal cortex threshold. Before and after the intervention, the Korean version of the occupational stress inventory (K-OSI), Beck's depression inventory (BDI), and Beck's anxiety inventory (BAI) were administered and EEG was performed using a 21-channel digital EEG system.
\end{abstract}

Results After TMS, the average scores for the affective responses to stressors on the personal strain questionnaire (PSQ) subscale of K-OSI and BDI decreased significantly for the active-TMS group compared to the sham-TMS group. Also, the active-TMS group showed a significantly greater decrease in relative alpha in the F3 electrode and a significantly greater increase in the F4 electrode.

Conclusion High-frequency rTMS on the left DLPFC had stress-relieving and mood-elevating effects in health care workers, likely by stimulating the left frontal lobe.

Psychiatry Investig 2016;13(6):622-629

Key Words Repetitive transcranial magnetic stimulation, Occupational stress, Quantitative electroencephalography, Health care workers.

\section{INTRODUCTION}

Occupational stress refers to the emotional stress that occurs in the course of performing duties in the workplace. It may due to a complex job description, role ambiguity, job insecurity, work overload, and/or unsafe working conditions. ${ }^{1}$ Occupational stress can also be caused by other difficulties in the course of duty, such as an interpersonal conflict in the workplace, an irrational or authoritative culture of the organization, or a conflict between work and family. ${ }^{2}$ Interest in occupational stress is rising because it can serve as a motiva-

Received: May 26, 2015 Revised: July 16, 2015

Accepted: July 29, 2015 Available online: November 3, 2015

$\triangle$ Correspondence: Sun Mi Kim, MD, PhD

Department of Psychiatry, Chung-Ang University Hospital, 102 Heukseok-ro, Dongjak-gu, Seoul 06973, Republic of Korea

Tel: +82-2-6299-1519, Fax: +82-2-6298-1508

E-mail: sunmikim706@gmail.com

(a) This is an Open Access article distributed under the terms of the Creative Commons Attribution Non-Commercial License (http://creativecommons.org/licenses/bync/3.0) which permits unrestricted non-commercial use, distribution, and reproduction in any medium, provided the original work is properly cited. tion for drinking, smoking, or other activities that negatively impact the health of workers. ${ }^{2}$ In addition, it can cause negative emotions such as depression and anxiety, which can bring personal harm and social costs. ${ }^{3}$

Health care workers are known to experience high levels of occupational stress because they are repeatedly exposed to critical and emergency events due to the nature of hospital work. ${ }^{4}$ Furthermore, they are expected to have high professional competence and moral standards, and are also expected to be able to accomplish excessive work. Physicians usually work long hours and experience many occupational stresses, which results in a high prevalence of depression among them. ${ }^{5}$ Among professionals in a hospital, nurses are also known to be more depressed and mentally exhausted compared with general workers. ${ }^{6}$ In fact, most clinical health services are delivered by nurses and nurses are in direct contact with patients most of the time. With the recent emphasis on services for customer satisfaction, health care professionals are expected to not only restore the physical stability of patients, 
but to come across as tender, caring, and empathetic. ${ }^{4}$

Previous studies have reported that occupational stress induces mental health problems. ${ }^{7-9}$ It was shown that teachers with high occupational stress reported a decreased level of perceived quality of life; however, adequate stress management through social support could improve their quality of life. ${ }^{9}$ In another study, fire officials with high subjective occupational satisfaction showed lower levels of depression compared to those with low subjective occupational satisfaction. ${ }^{8}$ Moreover, distress in the job environment was a significant predictor of depression among Korean workers.?

Repetitive transcranial magnetic stimulation (rTMS) is a therapeutic technique for applying stimulation to the cerebral cortex in a non-invasive manner. ${ }^{10} \mathrm{~A}$ current in the coil of the TMS equipment generates a rapidly changing external magnetic field that, in turn, induces an electric current in nerve cells in the brain, thus activating neurons. TMS can regulate the cortical excitability by varying frequency; high-frequency TMS increases cortical excitability, while low-frequency TMS decreases cortical excitability. ${ }^{11} \mathrm{rTMS}$ is used in the treatment of various psychiatric and neurologic diseases such as depression, post-traumatic stress disorder (PTSD), social anxiety disorder, schizophrenia, and Parkinson's disease, as well as in neuro-rehabilitation. ${ }^{10,12-15}$ rTMS was approved by the Food and Drug Administration (FDA) as a treatment to alleviate symptoms of treatment-resistant depression. ${ }^{13}$ A method of TMS that is effective for depressed patients is high-frequency stimulation on the left dorsolateral prefrontal cortex (DLPFC). In a previous study, it was shown that high-frequency rTMS over the left DLPFC caused a reduction in alpha activity and an up-regulation in cortical activity in depressed patients. ${ }^{16} \mathrm{In}$ addition, the decrease in alpha activity was significantly associated with a reduction in the severity of clinical symptoms.

Unfortunately, so far, there are only limited options in the treatment of occupational stress, including both pharmacological and non-pharmacological interventions. Pharmacological treatment has been used for management of psychiatric symptoms caused by acute and chronic occupational stress, such as depressed mood, anxiety, insomnia, and substance dependence. ${ }^{17}$ Non-pharmacological interventions have been used for improving coping strategies and relaxing, including cognitive behavior therapy, relaxation therapy, art therapy, as well as psychotherapy. ${ }^{18}$ Although non-pharmacological interventions are widely used for occupational stress in the clinical setting, there are still very few reports of well-designed randomized controlled trials on their effectiveness. With this study, we aimed to investigate whether rTMS could be an effective non-pharmacological treatment option for occupational stress. We expected that rTMS could be a suitable and effective treatment option, especially for patients who would prefer not to take medications or who experienced adverse effects of pharmacological treatment.

\section{Hypothesis}

The effects of rTMS on occupational stress have not yet been conducted in spite of the close relationship between job stress, burnout, and depression. We hypothesized that highfrequency rTMS delivered to the left DLPFC would produce significant stress-relieving and mood-elevating effects among health care workers. We also hypothesized that rTMS would cause a decrease in relative alpha in the left frontal lobe when measured by quantitative electroencephalography (QEEG).

\section{METHODS}

\section{Study participants}

Health care workers in four university hospitals in Seoul were recruited with an advertisement in hospital bulletins. Twenty-eight healthy adult women, aged 24 to 45 , were recruited. The exclusion criteria were as follows: individuals with 1) past or current diagnosis of any axis I psychiatric disorder based on the Structured Clinical Interview for DSM-IV-TR Axis I Disorders, Patient Edition (SCID-I/P), ${ }^{19} 2$ ) severe medical illness, 3) organic mental disorder, seizure disorder, or mental retardation, 4) pregnancy, 5) current psychotropic medication use, 6) surgical treatment of intracranial lesions, or 7) a magnetic substance in their brain or orbital area. The Institutional Review Board at the Chung-Ang University Hospital approved the study protocol, and all participants provided written informed consent. All study procedures including screening participants, baseline and follow-up assessments of clinical scales and EEG, and data analyses were conducted at the Chung-Ang University Hospital.

At baseline and at the end of the study, patients were assessed with the Korean version of the occupational stress inventory (K-OSI), ${ }^{20,21}$ Beck's depression inventory (BDI), ${ }^{22}$ Beck's anxiety inventory (BAI), ${ }^{23}$ and QEEG using a 21-channel digital EEG system. Participants were randomly divided into two groups: the active-TMS group and the sham-TMS group. Among the 28 enrolled participants, data from four participants were dropped because they did not complete the TMS sessions or QEEG assessment: one participant from the active-TMS group and one participant from the sham-TMS group discontinued the TMS sessions due to headache and one participant from the active-TMS group and one participant from the sham-TMS group missed their QEEG appointments without giving notification. Ultimately, 24 participants completed all TMS sessions and QEEG assessments. 


\section{Repetitive transcranial magnetic stimulation procedures}

rTMS was performed with TAMAS (CR Technology, Korea). All participants underwent 12 sessions of active or sham rTMS delivered to the DLPFC three times a week for four weeks. The standardized treatment location was over the left prefrontal cortex, defined as $5 \mathrm{~cm}$ anterior to the motor threshold location along a left superior oblique plane with a rotation point about the tip of the patient's nose. ${ }^{24}$ The motor threshold was estimated before each treatment, which was defined as the lowest stimulation power capable of inducing movement in the right abductor pollicis brevis muscle. With a descending method of limits procedure with $2 \%$ steps, the motor threshold was defined as the minimal intensity that produced right thumb contraction. Each session consisted of 32 trains of $10 \mathrm{~Hz}$ repetitive TMS delivered in 5-second trains at $110 \%$ of the estimated motor threshold. Sham-TMS was performed with a two-wing $90^{\circ}$ tilt - sham (placebo) condition..$^{25}$

\section{Measures}

The Korean version of the Occupational Stress Inventory

We used K-OSI ${ }^{20}$ which was translated into Korean and standardized by Lee et al. ${ }^{21}$ to assess the level of occupational stress of the participants. K-OSI is a multidimensional as- sessment that consists of 14 sub-measures in three areas (Table 1): 1) stress factors in the workplace: occupational role questionnaire (ORQ), 2) affective responses to stressors: personal strain questionnaire (PSQ), and 3) coping resources for occupational stress: personal resource questionnaire (PRQ). The ORQ consisted of six subscales: role overload (RO), role insufficiency (RI), role ambiguity (RA), role boundary (RB), responsibility (R), and physical environment $(\mathrm{PE})$. The PSQ consisted of four subscales: vocational strain (VS), psychological strain (PSY), interpersonal strain (IS), and physical strain (PHS). Lastly, the PRQ consisted of four subscales: recreation (RE), self-care (SC), social support (SS), and rational/ cognitive coping $(\mathrm{RC})$.

Beck's depression inventory and Beck's anxiety inventory $\mathrm{BDI}^{22}$ was used to assess the participants' level of depression symptoms during the past two weeks. $\mathrm{BAI}^{23}$ was used to assess the participants' level of anxiety during the past week.

\section{Quantitative electroencephalography data acquisition and analysis \\ Electroencephalographic (EEG) activity was recorded with a 21-channel EEG system (model CMXL-P230, Grass-Tele- factor, West Warwick, RI, USA). The electrodes were placed on the scalp according to the International 10-20 System with}

Table 1. The questionnaires used in this study and the 14 subscales of the Korean version of the Occupational Stress Inventory

\begin{tabular}{|c|c|}
\hline Questionnaires and subscales & Measures \\
\hline Occupational role questionnaire & Measures stress factors in the workplace \\
\hline Role overload & $\begin{array}{l}\text { Measures the degree to which a person can perform the job and the degree to which the demand } \\
\text { of the job exceeds the individual's ability or environmental conditions }\end{array}$ \\
\hline Role insufficiency & $\begin{array}{l}\text { Measures whether the skill training and experience level of the worker are appropriate for the } \\
\text { job role }\end{array}$ \\
\hline Role ambiguity & Measures the compatibility of task priority, task expectation, and evaluation criteria \\
\hline Role boundary & Measures the degree to which the employee experiences inappropriate role demands \\
\hline Responsibility & $\begin{array}{l}\text { Measures the degree to which the person feels responsible for the job performance and welfare } \\
\text { of others }\end{array}$ \\
\hline Physical environment & Measures the degree of exposure to a hazardous working environment \\
\hline Personal strain questionnaire & Measures the affective responses to stressors \\
\hline Vocational strain & Measures the grievances and attitude related to the work quality or production \\
\hline Psychological strain & Measures the degree of emotional and psychological difficulty \\
\hline Interpersonal strain & Measures the degree of conflict and difficulty of interpersonal relationships \\
\hline Physical strain & Measures physical illness and self-care habits related to poor health \\
\hline Personal resource questionnaire & Measures coping resources for occupational stress \\
\hline Recreation & Measures the degree of relaxation and joy through activities performed for pleasure \\
\hline Self-care & $\begin{array}{l}\text { Measures the degree to which regular self-management activities that address chronic stress are } \\
\text { effective }\end{array}$ \\
\hline Social support & $\begin{array}{l}\text { Measures the extent to which the person feels that they receive support and help from other } \\
\text { people }\end{array}$ \\
\hline Rational/cognitive coping & Measures the degree to which cognitive skills help cope with stress related to the job \\
\hline
\end{tabular}


two additional electrodes placed on the inter-connected ear lobe to serve as the reference (A2) and ground (A1) electrodes. The QEEG was recorded with a resolution of 12 bits, a lowfrequency filter of $0.5 \mathrm{~Hz}$, a high-frequency filter of $46 \mathrm{~Hz}$, and a sampling frequency of $256 \mathrm{~Hz}$. Impedance was maintained below $5 \mathrm{k} \Omega$. All recordings were performed by the same EEG technician in an electro-magnetically shielded room in the Chung-Ang University Hospital.

EEG activity was recorded with open eyes for 5 minutes and closed eyes for 5 minutes. Artifact-free 120 -second periods from the eyes-open condition and 120-second periods from the eyes-closed condition were selected for analysis. Epochs of movement-related artifacts were excluded from the analyses by direct visual inspection of the raw data. The results are presented as absolute spectral power values $\left(\mu V^{2} / \mathrm{Hz}\right)$ for individual segments of the EEG spectra: delta $(0.5-4 \mathrm{~Hz})$, theta $(4-8 \mathrm{~Hz})$, alpha $(8-12 \mathrm{~Hz})$, sigma $(12-16 \mathrm{~Hz})$, low beta
(16-24 Hz), high beta $(24-32 \mathrm{~Hz})$, and gamma $(32-50 \mathrm{~Hz})$. The average power spectrum of the EEG frequencies was calculated by fast Fourier transform (FFT), a mathematical process that identifies the various frequency bands in the QEEG data. Relative power values for each band were derived by expressing absolute power in each frequency band as a percent of the absolute power summed over seven frequency bands (0.5-50 Hz). Complexity 2.0 (Laxtha, Inc., Daejeon, Korea) EEG was used to analyze the QEEG data.

\section{Statistical analysis}

The differences in age, years of education, duration of employment, working hours per week, marital status, occupation, socioeconomic status, K-OSI scores, BDI scores, and BAI scores between the active-TMS group and the sham-TMS group were analyzed using independent t-tests and chi-square tests. The changes in mean scores of clinical scales from base-

Table 2. General demographic and clinical characteristics of the study population

\begin{tabular}{|c|c|c|c|}
\hline Characteristic & Active-TMS $(\mathrm{N}=12)$ & Sham-TMS $(\mathrm{N}=12)$ & Statistics \\
\hline$\overline{\text { Age }}$ & $28.0 \pm 3.0$ & $31.9 \pm 7.1$ & $\mathrm{t}=1.76, \mathrm{p}=0.10$ \\
\hline Years of education & $15.7 \pm 1.2$ & $14.3 \pm 1.4$ & $\mathrm{t}=-2.51, \mathrm{p}<0.05$ \\
\hline Duration of employment (years) & $4.3 \pm 2.5$ & $8.9 \pm 7.0$ & $\mathrm{t}=2.16, \mathrm{p}=0.05$ \\
\hline Working hours per week & $48.8 \pm 14.9$ & $48.3 \pm 6.2$ & $\mathrm{t}=-0.13, \mathrm{p}=0.90$ \\
\hline Marital status (married/unmarried) & $4 / 8$ & $4 / 8$ & $\chi^{2}=0.00, p=1.00$ \\
\hline Occupation (doctor/nurse/other) & $4 / 4 / 4$ & $1 / 9 / 2$ & $\chi^{2}=4.39, p=0.11$ \\
\hline Socioeconomic status (high/middle/low) & $0 / 9 / 3$ & $1 / 8 / 3$ & $\chi^{2}=1.06, p=0.59$ \\
\hline \multicolumn{4}{|l|}{ K-OSI } \\
\hline ORQ & $322.8 \pm 19.9$ & $333.4 \pm 21.7$ & $\mathrm{t}=1.24, \mathrm{p}=0.23$ \\
\hline $\mathrm{RO}$ & $54.2 \pm 6.9$ & $53.3 \pm 6.6$ & $\mathrm{t}=-0.33, \mathrm{p}=0.74$ \\
\hline RI & $50.3 \pm 5.6$ & $54.0 \pm 6.3$ & $\mathrm{t}=1.51, \mathrm{p}=0.15$ \\
\hline RA & $52.3 \pm 8.5$ & $54.4 \pm 9.8$ & $\mathrm{t}=0.58, \mathrm{p}=0.57$ \\
\hline $\mathrm{RB}$ & $54.6 \pm 5.5$ & $57.7 \pm 6.2$ & $\mathrm{t}=1.30, \mathrm{p}=0.21$ \\
\hline $\mathrm{R}$ & $58.1 \pm 7.0$ & $56.0 \pm 6.2$ & $\mathrm{t}=-0.77, \mathrm{p}=0.45$ \\
\hline $\mathrm{PE}$ & $53.4 \pm 10.7$ & $58.1 \pm 8.4$ & $\mathrm{t}=1.19, \mathrm{p}=0.25$ \\
\hline PSQ & $242.8 \pm 28.1$ & $223.7 \pm 25.1$ & $\mathrm{t}=-1.75, \mathrm{p}=0.09$ \\
\hline VS & $60.3 \pm 9.7$ & $52.6 \pm 10.3$ & $\mathrm{t}=-1.87, \mathrm{p}=0.07$ \\
\hline PSY & $64.9 \pm 10.3$ & $60.4 \pm 7.8$ & $\mathrm{t}=-1.20, \mathrm{p}=0.24$ \\
\hline IS & $56.3 \pm 5.7$ & $54.3 \pm 6.8$ & $\mathrm{t}=-0.75, \mathrm{p}=0.46$ \\
\hline PHS & $61.3 \pm 10.2$ & $56.3 \pm 8.6$ & $\mathrm{t}=-1.30, \mathrm{p}=0.21$ \\
\hline PRQ & $179.2 \pm 21.7$ & $178.5 \pm 21.8$ & $\mathrm{t}=-0.08, \mathrm{p}=0.94$ \\
\hline $\mathrm{RE}$ & $49.1 \pm 6.1$ & $44.8 \pm 9.9$ & $\mathrm{t}=-1.29, \mathrm{p}=0.21$ \\
\hline SC & $40.5 \pm 6.3$ & $43.4 \pm 8.5$ & $\mathrm{t}=0.95, \mathrm{p}=0.35$ \\
\hline SS & $44.8 \pm 9.7$ & $46.3 \pm 9.4$ & $\mathrm{t}=0.41, \mathrm{p}=0.69$ \\
\hline $\mathrm{RC}$ & $44.8 \pm 9.5$ & $44.0 \pm 9.9$ & $\mathrm{t}=-0.21, \mathrm{p}=0.84$ \\
\hline BDI & $11.3 \pm 6.7$ & $10.4 \pm 6.5$ & $\mathrm{t}=-0.31, \mathrm{p}=0.76$ \\
\hline BAI & $8.5 \pm 5.5$ & $9.5 \pm 8.5$ & $\mathrm{t}=0.34, \mathrm{p}=0.74$ \\
\hline
\end{tabular}

K-OSI: the Korean version of the Occupational Stress Inventory, ORQ: occupational role questionnaire, RO: role overload, RI: role insufficiency, RA: role ambiguity, RB: role boundary, R: responsibility, PE: physical environment, PSQ: personal strain questionnaire, VS: vocational strain, PSY: psychological strain, IS: interpersonal strain, PHS: physical strain, PRQ: personal resource questionnaire, RE: recreation, SC: self-care, SS: social support, RC: rational/cognitive coping, BDI: Beck's depression inventory, BAI: Beck's anxiety inventory 
line to the follow-up assessment were investigated using repeated measures analysis of covariance (ANCOVA) in both groups controlling for age, years of education, duration of employment, and working hours per week. The changes in relative alpha power in the frontal area were analyzed using repeated measures ANCOVA in both groups controlling for age, years of education, duration of employment, working hours per week, BDI score, and BAI score. All analyses were performed using Statistica 6.0 (2001, StatSoft, Tulsa, OK, USA).

\section{RESULTS}

\section{Demographic and clinical characteristics}

At baseline, there were no significant differences between the active-TMS group and the sham-TMS group in regard to age, duration of employment, working hours per week, marital status, occupation, socioeconomic status, all subscales of K-OSI scores, BDI score, and BAI score (Table 2). The activeTMS group showed a significantly higher mean number of years of education $(\mathrm{t}=-2.51, \mathrm{p}<0.05)$.

\section{Changes in mean scores of clinical scales from baseline to follow-up}

During the four-week intervention period, the active-TMS group showed significantly greater reductions on the VS $(\mathrm{F}=5.16, \mathrm{p}<0.05)$ (Table 3), PSY $(\mathrm{F}=13.92, \mathrm{p}<0.01)$, and PHS $(\mathrm{F}=11.30, \mathrm{p}<0.01)$ subscale scores of PSQ in addition to the total PSQ score $(\mathrm{F}=20.14, \mathrm{p}<0.001)$ on the K-OSI. The activeTMS group also showed significantly greater decreases in the BDI score $(\mathrm{F}=8.70, \mathrm{p}<0.01)$ compared to the sham-TMS group. There was no significant difference between the active-TMS and the sham-TMS groups in regard to the change in the total ORQ and PRQ scores and the subscale scores of those questionnaires on the K-OSI, as well as the BAI.

\section{Changes in relative alpha power in the frontal area}

During the four-week intervention period, the active-TMS group showed a significantly greater decrease in relative alpha in the F3 electrode $(\mathrm{F}=5.50, \mathrm{p}<0.05)$ (Table 4, Figure 1) and a significantly greater increase in the $\mathrm{F} 4$ electrode $(\mathrm{F}=$ $6.62, \mathrm{p}<0.05)$.

Table 3. Changes in mean scores of clinical scales from the baseline to the follow-up assessment

\begin{tabular}{|c|c|c|c|c|c|c|}
\hline & \multicolumn{2}{|c|}{ Active-TMS $(\mathrm{N}=12)$} & \multicolumn{2}{|c|}{ Sham TMS $(\mathrm{N}=12)$} & \multicolumn{2}{|c|}{ Repeated measures ANCOVA } \\
\hline & Baseline & Follow-up & Baseline & Follow-up & Group $\times$ visit effect & Effect size $\left(\eta_{p}^{2}\right)$ \\
\hline \multicolumn{7}{|l|}{ K-OSI } \\
\hline ORQ & $322.8 \pm 19.9$ & $321.6 \pm 20.6$ & $333.4 \pm 21.7$ & $339.9 \pm 27.6$ & $\mathrm{~F}=0.05, \mathrm{p}=0.83$ & 0.003 \\
\hline $\mathrm{RO}$ & $54.2 \pm 6.9$ & $52.3 \pm 3.2$ & $53.3 \pm 6.6$ & $54.0 \pm 7.1$ & $\mathrm{~F}=0.50, \mathrm{p}=0.49$ & 0.027 \\
\hline RI & $50.3 \pm 5.6$ & $48.5 \pm 4.3$ & $54.0 \pm 6.3$ & $52.9 \pm 5.1$ & $\mathrm{~F}=0.06, \mathrm{p}=0.81$ & 0.003 \\
\hline RA & $52.3 \pm 8.5$ & $51.3 \pm 8.6$ & $54.4 \pm 9.8$ & $55.5 \pm 6.8$ & $\mathrm{~F}=0.03, \mathrm{p}=0.86$ & 0.002 \\
\hline $\mathrm{RB}$ & $54.6 \pm 5.5$ & $55.0 \pm 4.9$ & $57.7 \pm 6.2$ & $60.3 \pm 6.0$ & $\mathrm{~F}=0.19, \mathrm{p}=0.67$ & 0.010 \\
\hline $\mathrm{R}$ & $58.1 \pm 7.0$ & $58.5 \pm 6.7$ & $56.0 \pm 6.2$ & $57.3 \pm 9.1$ & $\mathrm{~F}=0.09, \mathrm{p}=0.77$ & 0.005 \\
\hline $\mathrm{PE}$ & $53.4 \pm 10.7$ & $56.0 \pm 9.0$ & $58.1 \pm 8.4$ & $60.0 \pm 7.1$ & $\mathrm{~F}=0.21, \mathrm{p}=0.65$ & 0.012 \\
\hline PSQ & $242.8 \pm 28.1$ & $216.5 \pm 23.0$ & $223.7 \pm 25.1$ & $240.6 \pm 17.5$ & $\mathrm{~F}=20.14, \mathrm{p}<0.001$ & 0.528 \\
\hline VS & $60.3 \pm 9.7$ & $56.6 \pm 9.5$ & $52.6 \pm 10.3$ & $61.8 \pm 9.9$ & $\mathrm{~F}=5.16, \mathrm{p}<0.05$ & 0.223 \\
\hline PSY & $64.9 \pm 10.3$ & $56.7 \pm 7.3$ & $60.4 \pm 7.8$ & $63.0 \pm 8.0$ & $\mathrm{~F}=13.92, \mathrm{p}<0.01$ & 0.436 \\
\hline IS & $56.3 \pm 5.7$ & $53.0 \pm 7.0$ & $54.3 \pm 6.8$ & $56.5 \pm 6.2$ & $\mathrm{~F}=3.36, \mathrm{p}=0.08$ & 0.157 \\
\hline PHS & $61.3 \pm 10.2$ & $50.3 \pm 7.5$ & $56.3 \pm 8.6$ & $59.3 \pm 9.3$ & $\mathrm{~F}=11.30, \mathrm{p}<0.01$ & 0.386 \\
\hline PRQ & $179.2 \pm 21.7$ & $192.2 \pm 17.9$ & $178.5 \pm 21.8$ & $185.0 \pm 22.5$ & $\mathrm{~F}=1.11, \mathrm{p}=0.31$ & 0.058 \\
\hline $\mathrm{RE}$ & $49.1 \pm 6.1$ & $50.2 \pm 9.1$ & $44.8 \pm 9.9$ & $48.8 \pm 9.9$ & $\mathrm{~F}=0.09, \mathrm{p}=0.77$ & 0.005 \\
\hline $\mathrm{SC}$ & $40.5 \pm 6.3$ & $45.8 \pm 7.8$ & $43.4 \pm 8.5$ & $43.9 \pm 7.9$ & $\mathrm{~F}=3.53, \mathrm{p}=0.08$ & 0.164 \\
\hline SS & $44.8 \pm 9.7$ & $48.1 \pm 5.2$ & $46.3 \pm 9.4$ & $44.6 \pm 10.4$ & $\mathrm{~F}=1.86, \mathrm{p}=0.19$ & 0.094 \\
\hline $\mathrm{RC}$ & $44.8 \pm 9.5$ & $48.2 \pm 6.6$ & $44.0 \pm 9.9$ & $47.7 \pm 7.5$ & $\mathrm{~F}=0.05, \mathrm{p}=0.83$ & 0.003 \\
\hline BDI & $11.3 \pm 6.7$ & $6.9 \pm 4.8$ & $10.4 \pm 6.5$ & $8.9 \pm 7.1$ & $\mathrm{~F}=8.70, \mathrm{p}<0.01$ & 0.326 \\
\hline BAI & $8.5 \pm 5.5$ & $4.4 \pm 4.0$ & $9.5 \pm 8.5$ & $8.9 \pm 7.0$ & $\mathrm{~F}=2.15, \mathrm{p}=0.16$ & 0.107 \\
\hline
\end{tabular}

A repeated measure ANCOVA was conducted controlling for age, years of education, duration of employment, and working hours per week. $\eta_{\mathrm{p}}^{2}$ : Partial Eta squared, TMS: transcranial magnetic stimulation, K-OSI: the Korean version of the Occupational Stress Inventory, ORQ: occupational role questionnaire, RO: role overload, RI: role insufficiency, RA: role ambiguity, RB: role boundary, R: responsibility, PE: physical environment, PSQ: personal strain questionnaire, VS: vocational strain, PSY: psychological strain, IS: interpersonal strain, PHS: physical strain, PRQ: personal resource questionnaire, RE: recreation, SC: self-care, SS: social support, RC: rational/cognitive coping, BDI: Beck's depression inventory, BAI: Beck's anxiety inventory 
Table 4. Changes in frontal alpha waves from baseline to the follow-up assessment

\begin{tabular}{|c|c|c|c|c|c|c|}
\hline & \multicolumn{2}{|c|}{ Active-TMS $(\mathrm{N}=12)$} & \multicolumn{2}{|c|}{ Sham-TMS $(\mathrm{N}=12)$} & \multicolumn{2}{|c|}{ Repeated measures ANCOVA } \\
\hline & Baseline & Follow-up & Baseline & Follow-up & Group $\times$ visit effect & Effect size $\left(\eta_{P}^{2}\right)$ \\
\hline Alpha Fp1-A1 & $0.031 \pm 0.029$ & $0.032 \pm 0.026$ & $0.026 \pm 0.022$ & $0.041 \pm 0.038$ & $\mathrm{~F}=0.78, \mathrm{p}=0.39$ & 0.046 \\
\hline Alpha Fp2-A2 & $0.026 \pm 0.022$ & $0.037 \pm 0.036$ & $0.031 \pm 0.028$ & $0.038 \pm 0.031$ & $\mathrm{~F}=0.00, \mathrm{p}=1.00$ & 0.000 \\
\hline Alpha F3-A1 & $0.141 \pm 0.129$ & $0.098 \pm 0.073$ & $0.091 \pm 0.088$ & $0.135 \pm 0.069$ & $\mathrm{~F}=5.50, \mathrm{p}<0.05$ & 0.256 \\
\hline Alpha F4-A2 & $0.093 \pm 0.077$ & $0.154 \pm 0.098$ & $0.156 \pm 0.126$ & $0.123 \pm 0.101$ & $\mathrm{~F}=6.62, \mathrm{p}<0.05$ & 0.293 \\
\hline Alpha F7-A1 & $0.057 \pm 0.048$ & $0.068 \pm 0.049$ & $0.082 \pm 0.079$ & $0.078 \pm 0.073$ & $\mathrm{~F}=0.09, \mathrm{p}=0.76$ & 0.006 \\
\hline Alpha F8-A2 & $0.075 \pm 0.058$ & $0.079 \pm 0.050$ & $0.097 \pm 0.088$ & $0.092 \pm 0.092$ & $\mathrm{~F}=0.00, \mathrm{p}=1.00$ & 0.000 \\
\hline Alpha Fz-A1 & $0.099 \pm 0.091$ & $0.166 \pm 0.093$ & $0.150 \pm 0.132$ & $0.125 \pm 0.100$ & $\mathrm{~F}=3.92, \mathrm{p}=0.07$ & 0.197 \\
\hline
\end{tabular}

Controlling for age, years of education, duration of employment, working hours per week, BDI score, and BAI score, a repeated measure ANCOVA was conducted to show the changes in alpha waves between baseline and follow-up. TMS: transcranial magnetic stimulation, BDI: Beck's depression inventory, BAI: Beck's anxiety inventory, $\eta_{\mathrm{p}}^{2}$ : Partial Eta squared

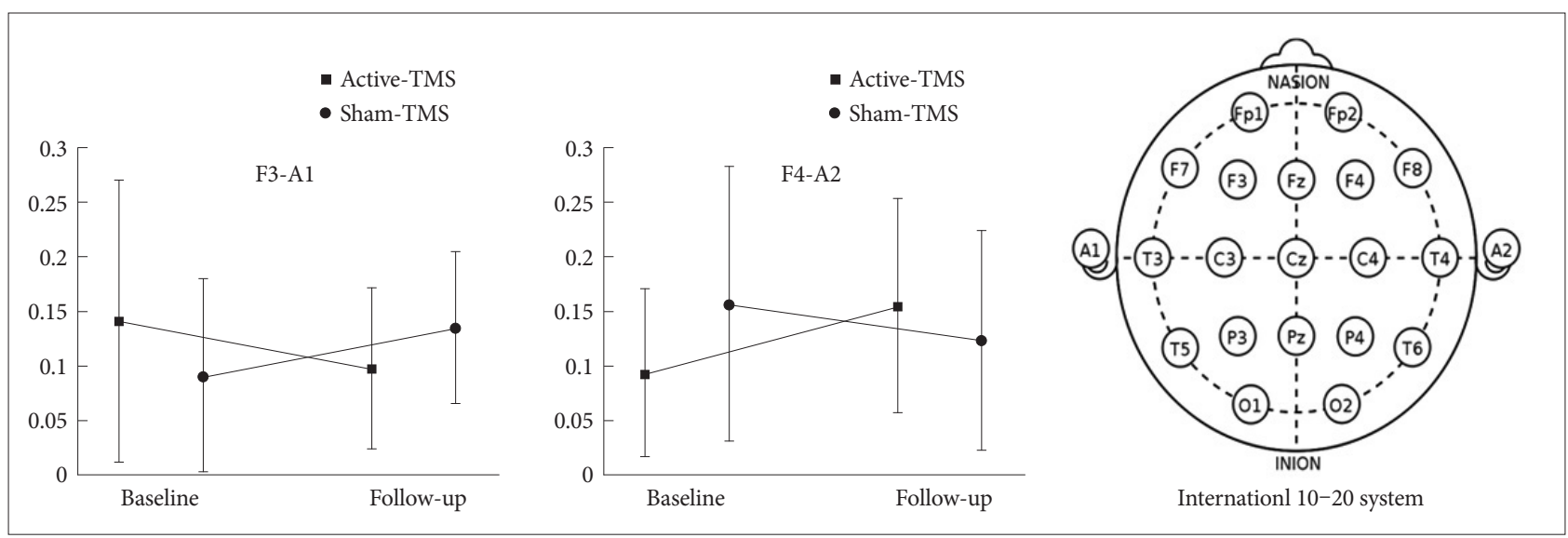

Figure 1. Changes in relative alpha power in the frontal area from baseline to the follow-up assessment. TMS: transcranial magnetic stimulation.

\section{DISCUSSION}

The most significant findings of this study were that TMS caused a significant decrease in scores on the PSQ subscale of the K-OSI and the BDI. Also, the active-TMS group showed a significantly greater decrease in the relative alpha in the F3 electrode and a significantly greater increase in the F4 electrode compared to the sham-TMS group. High-frequency rTMS on the left DLPFC seemed to stimulate the left frontal lobe and cause a decrease in the relative alpha, resulting in stress-relieving and mood-elevating effects.

In this study, rTMS produced significant stress-relieving and mood-elevating effects among health care workers. The PSQ subscale of the K-OSI reflects affective responses to stressors. Occupational stress is burdensome, can put pressure on individuals, and can cause tension among colleagues. ${ }^{1}$ Risks inherent with occupational stress are known to confer a negative influence on the quality of life and health status of workers. ${ }^{3}$ Although we were not able to control the stress factors in the workplace per se, rTMS reduced the grievances and improved the attitude related to the occupation (measured by VS), reduced the degree of emotional and psychological difficulty (measured by PSY), reduced the physical illness and self-care habits related to poor health (measured by PHS), as well as reduced the severity of depressive symptoms (measured by BDI).

In this study, we applied high-frequency rTMS to the left DLPFC to the participants in the active-TMS group. This protocol was similar to the protocol that was approved by the FDA as an alternative treatment for treatment-resistant depression. The left DLPFC is a critical area for depression because focal blood flow in the left DLPFC decreases in depressed patients and, accordingly, the prevalence of depression increases in patients with left prefrontal lobe infarcts. ${ }^{26}$ In particular, the functional connectivity between the DLPFC and the thalamus in treatment-resistant patients is known to be lessened. ${ }^{27}$ Therefore, we cautiously suggest that rTMS may lessen negative affect and reduce psychological depression caused by occupational stress by a mechanism similar to how rTMS alleviates treatment-resistant depression. Interestingly, self-perception of physical health was also improved by rTMS in this study. Occupational stress is known to directly cause physical pain and burnout. In fact, musculoskeletal symptoms, especially pain, are associated with not only intensity of labor but also occupational stress. ${ }^{1}$ rTMS may be able to reduce the occupational stress-induced negative affect and lead 
to improved self-perception of physical health.

According to the QEEG results of this study, rTMS caused a greater decrease in relative alpha in the left frontal lobes. Major depressive disorders may be related to hypo-activity in the left prefrontal cortex, which means decreased metabolism and dysfunction in the relevant cortical region. ${ }^{28} \mathrm{Hypo}$-activity in the left frontal lobe is a marker for depression because the alpha idling rhythm in this area may result in a deficit of positive emotions. Left prefrontal hypo-activation can manifest as an increase of alpha power in QEEG, which has an inverse association with neural excitability. ${ }^{16}$ Thus, we cautiously suggest that high-frequency rTMS on the left DLPFC has stress-relieving and mood-elevating effects by stimulating the left frontal lobe.

\section{Limitations}

The current study had some limitations. First, the number of participants was not large enough to generalize the effects of rTMS on occupational stress to all health care workers; future studies with a larger number of participants will be needed. Second, the clinicians were not blinded to the TMS group allocation and, thus, it is unknown if observer bias occurred; a double-blind research method could avoid this problem in the future. Finally, because we only recruited health care workers, it was not possible to comment on the level of occupational stress these participants were experiencing relative to the general population.

\section{Conclusion}

High-frequency rTMS on the left DLPFC had stress-relieving and mood-elevating effects, and these effects were likely due to stimulation of the left frontal lobe. Future studies with a larger population and a longer follow-up period are needed to generalize our results to a broader patient base and assess the optimal amount of time for the treatment.

\section{Acknowledgments}

This work was supported by a grant from the Korea Creative Content Agency (R2014040055).

\section{REFERENCES}

1. Kim H, June K, Shin G, Choo J. Associations between job stress and work-related musculoskeletal symptoms in street sanitation workers. Korean Acad Commun Health Nurs 2013;24:314-322.

2. Rusli BN, Edimansyah BA, Naing L. Working conditions, self-perceived stress, anxiety, depression and quality of life: a structural equation modelling approach. BMC Public Health 2008;8:48.

3. Chang S, Koh S, Kang D, Kim S, Kang M, Lee C. Developing an occupational stress scale for Korean employees. Korean J Occup Environ Med 2005;17:297-317.

4. Kim Y. The Influence of a general hospital nurse's emotional labor, emotional intelligence on job stress. J Digit Convergence 2014;12:245-253.

5. Tomioka K, Morita N, Saeki K, Okamoto N, Kurumatani N. Working hours, occupational stress and depression among physicians. Occup Med (Lond) 2011;61:163-170.

6. Yoshizawa K, Sugawara N, Yasui-Furukori N, Danjo K, Furukori H, Sato Y, et al. Relationship between occupational stress and depression among psychiatric nurses in Japan. Arch Environ Occup Health 2014. [Epub ahead of print]

7. Cho JJ, Kim JY, Chang SJ, Fiedler N, Koh SB, Crabtree BF, et al. Occupational stress and depression in Korean employees. Int Arch Occup Environ Health 2008;82:47-57.

8. Kim K, Park J, Park B. The effects of job stress with depression and fatigue of firemen. J Korea Cont Assoc 2014;14:223-231.

9. Yang X, Ge C, Hu B, Chi T, Wang L. Relationship between quality of life and occupational stress among teachers. Public Health 2009;123: 750-755.

10. Aleman A. Use of repetitive transcranial magnetic stimulation for treatment in psychiatry. Clin Psychopharmacol Neurosci 2013;11:53-59.

11. Li CT, Wang SJ, Hirvonen J, Hsieh JC, Bai YM, Hong CJ, et al. Antidepressant mechanism of add-on repetitive transcranial magnetic stimulation in medication-resistant depression using cerebral glucose metabolism. J Affect Disord 2010;127:219-229.

12. Karsen EF, Watts BV, Holtzheimer PE. Review of the effectiveness of transcranial magnetic stimulation for post-traumatic stress disorder. Brain Stimul 2014;7:151-157.

13. Nemeroff CB. Prevalence and management of treatment-resistant depression. J Clin Psychiatry 2007;68(Suppl 8):17-25.

14. Sandrini M, Cohen LG. Noninvasive brain stimulation in neurorehabilitation. Handb Clin Neurol 2013;116:499-524.

15. Zhu H, Lu Z, Jin Y, Duan X, Teng J, Duan D. Low-frequency repetitive transcranial magnetic stimulation on Parkinson motor function: a metaanalysis of randomised controlled trials. Acta Neuropsychiatr 2015;27: 82-89.

16. Pellicciari MC, Cordone S, Marzano C, Bignotti S, Gazzoli A, Miniussi $\mathrm{C}$, et al. Dorsolateral prefrontal transcranial magnetic stimulation in patients with major depression locally affects alpha power of REM sleep. Front Hum Neurosci 2013;7:433.

17. Madsen IE, Lange T, Borritz M, Rugulies R. Burnout as a risk factor for antidepressant treatment - a repeated measures time-to-event analysis of 2936 Danish human service workers. J Psychiatr Res 2015;65: 47-52.

18. Ruotsalainen JH, Verbeek JH, Marine A, Serra C. Preventing occupational stress in healthcare workers. Cochrane Database Syst Rev 2014; 12:CD002892.

19. First M, Spitzer R, Gibbon M, Williams J. Structured Clinical Interview for DSM-IV-TR axis I Disorders, Research Version, Patient Edition. New York: Biometrics Research, New York State Psychiatric Institute; 2002.

20. Osipow S, Spokane A. Occupational Stress Inventory: Manual Research Version. Odessa, FL: Psychological Assessment Resources; 1992.

21. Lee J, Byun J, Kim S, Lee H, Kim H. The impact of occupational stress on suicide ideation of subway drivers. Occup Environ Med 2014;71:A74.

22. Beck AT, Ward CH, Mendelson M, Mock J, Erbaugh J. An inventory for measuring depression. Arch Gen Psychiatry 1961;4:561-571.

23. Beck AT, Epstein N, Brown G, Steer RA. An inventory for measuring clinical anxiety: psychometric properties. J Consult Clin Psychol 1988; 56:893-897.

24. George MS, Lisanby SH, Avery D, McDonald WM, Durkalski V, Pavlicova $\mathrm{M}$, et al. Daily left prefrontal transcranial magnetic stimulation therapy for major depressive disorder: a sham-controlled randomized trial. Arch Gen Psychiatry 2010;67:507-516.

25. Lisanby SH, Gutman D, Luber B, Schroeder C, Sackeim HA. Sham TMS: intracerebral measurement of the induced electrical field and the induction of motor-evoked potentials. Biol Psychiatry 2001;49:460-463.

26. George MS, Avery D, Nahas Z, Molloy M, Oliver NC, Risch SC, et al. rTMS studies of mood and emotion. Electroencephalogr Clin Neurophysiol Suppl 1999;51:304-314. 
27. Lui S, Wu Q, Qiu L, Yang X, Kuang W, Chan RC, et al. Resting-state functional connectivity in treatment-resistant depression. Am J Psychiatry 2011;168:642-648.
28. Drevets WC. Functional anatomical abnormalities in limbic and prefrontal cortical structures in major depression. Prog Brain Res 2000; 126:413-431. 